\title{
Das Medizinprodukte-Vigilancesystem am Beispiel von Blutzuckermesssystemen
}

Andreas Schlegel

Korrespondenz:

Dr. phil. nat. Andreas Schlegel Swissmedic,

Schweizerisches Heilmittelinstitut Abteilung Medizinprodukte Hallerstrasse 7

Postfach

CH-3000 Bern 9

Tel. 0313227701

Fax 0313227646

andreas.schlegel@swissmedic.ch

\section{Zusammenfassung}

Blutzuckermesssysteme für Diabetiker werden mit zwei verschiedenen Messeinheiten für die Angabe der Glukosekonzentration angeboten: $\mathrm{mmol} / \mathrm{L}$ und $\mathrm{mg} / \mathrm{dL}$. Der numerische Messwert in $\mathrm{mmol} / \mathrm{L}$ ist $18 \mathrm{mal}$ tiefer als jener in $\mathrm{mg} / \mathrm{dL}$. Falls ein Blutzuckermesssystem auf die Einheit $\mathrm{mg} / \mathrm{dL}$ eingestellt ist und die angegebenen Messresultate fälschlicherweise als Werte in $\mathrm{mmol} / \mathrm{L}$ interpretiert werden, kann eine Fehltherapie mit schwerwiegenden Folgen für den Diabetiker eintreten. Das gesetzlich vorgeschriebene Überwachungssystem (Medizinproduktevigilance, kurz Materiovigilance) dient der Erkennung und Behebung solcher Risiken. Aufgrund von sich häufenden Materiovigilancemeldungen zum Risiko der Messeinheiten konnten gezielt risikomindernde Massnahmen eingeführt werden. Anhand dieses praktischen Beispiels wird der Nutzen des Meldesystems aufgezeigt. Die Materiovigilance funktioniert allerdings nur, wenn die beteiligten Parteien, insbesondere Spitäler und Ärzte, ihre Beobachtungen über schwerwiegende Vorkommnisse melden.

\section{Einleitung}

Medizinprodukte sind in der Medizin verwendete Instrumente, Systeme oder andere medizinisch-technische Gegenstände. Medizinprodukte sind spezifisch und anders als Arzneimittel reguliert. Zu den Medizinprodukten gehören z.B. Röntgenapparate, Herzschrittmacher, Kontaktlinsen und In-vitro-Diagnostika, wie z.B. Blutzuckermesssysteme [1].

Die Materiovigilance ist ein Überwachungssystem, mit dem fehlerhafte Medizinprodukte oder Risiken im Umgang mit solchen Produkten erfasst werden. Die Hersteller und Händler, die Anwender und die nationalen Behörden sind an der Materiovigilance beteiligt [2]. Das System dient der Erkennung und Eliminierung fehlerhafter Produkte und damit zusammenhängender Risiken, z.B. mittels Rückrufen und Warnschreiben an die Anwender (dear doctor letters). Professionelle Anwender und Hersteller sind von Gesetzes wegen verpflichtet, schwerwiegende Vorkommnisse mit Medizinprodukten an die zuständige Behörde zu melden. In der Schweiz hat

\section{Les lecteurs de glycémie \\ pour diabétiques comme \\ exemple de vigilance \\ des dispositifs médicaux}

Les lecteurs de glycémie pour diabétiques sont disponibles avec deux unités de mesure différentes pour l'indication de la concentration du glucose: $\mathrm{mmol} / \mathrm{L}$ et $\mathrm{mg} / \mathrm{dL}$. La valeur mesurée en $\mathrm{mmol} / \mathrm{L}$ est 18 fois inférieure à celle en $\mathrm{mg} / \mathrm{dL}$. Au cas où un lecteur de glycémie est réglé sur l'unité en $\mathrm{mg} / \mathrm{dL}$ et que le résultat de la mesure est interprété par erreur comme une valeur en $\mathrm{mmol} / \mathrm{L}$, une thérapie erronée suivie de conséquences sérieuses peut se produire pour le diabétique. Le système de surveillance stipulé dans la loi pour de tels lecteurs (vigilance des dispositifs médicaux ou matériovigilance) sert à la reconnaissance et à la réduction de tels risques. En raison des annonces accumulées de matériovigilance relatives aux risques liés à ces lecteurs, des mesures visant à la réduction des risques ont pu être introduites. En s'appuyant sur cet exemple, l'utilité du système d'annonce est mis en évidence. La matériovigilance fonctionne cependant seulement quand les parties concernées, en particulier les hôpitaux et les médecins, annoncent leurs observations sur les incidents graves.

die Meldung an Swissmedic zu erfolgen [1]. Der Hersteller muss das gemeldete Vorkommnis untersuchen und nötigenfalls eine Korrektur einleiten, z.B. einen Rückruf oder ein Warnschreiben an Anwender. Swissmedic überwacht die Untersuchungen und Schlussfolgerungen der Hersteller. Als zentrale Meldestelle sammelt Swissmedic die Informationen über Vorkommnisse, Rückrufe und Warnschreiben und tauscht solche Informationen auch mit ausländischen Behörden aus. 
Der vorliegende Artikel beschreibt anhand spezieller Vorfälle, die mit Blutzuckermesssystemen (BGM) für Diabetiker aufgetreten sind, wie das Materiovigilancesystem Risiken erkennen und beseitigen kann. Die Vorfälle betrafen den Umstand, dass der Blutzuckerwert gegenwärtig in zwei Messeinheiten (mmol/L und $\mathrm{mg} / \mathrm{dL}$ ) angegeben wird, die numerisch differieren. Nichtbeachten der Messeinheit hat zu Fehlinterpretationen, Fehltherapie und medizinischen Komplikationen geführt. Aufgrund sich häufen-

\section{Abbildung 1}

In der Zeitspanne von Januar 2002 bis Juni 2007 gingen bei Swissmedic 50 Meldungen zu Blutzuckermesssystemen für Diabetiker ein. Die Meldungen wurden retrospektiv in Gruppen ähnlicher Problematik eingeteilt und sind hier nach entsprechender Häufigkeit dargestellt.

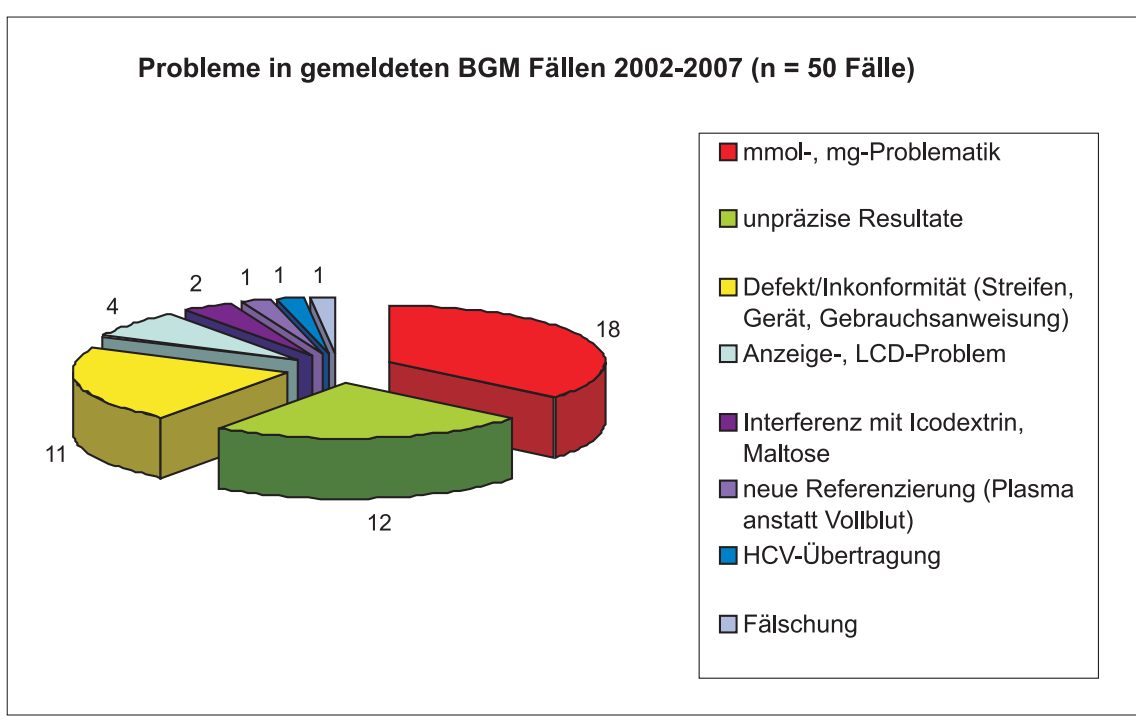

Abbildung 2

Die Frequenz aller eingegangenen Meldungen zu Blutzuckermesssystemen für Diabetiker wurde ermittelt. Die Frequenz der eingegangenen Meldungen zur mmol-/mg-Problematik, eine Teilmenge aller Meldungen, ist zum Vergleich aufgetragen. Ebenso die Teilmenge der Meldungen zu unpräzisen Resultaten. Die entsprechenden Werte für 2007 wurden anhand der Halbjahresdaten (Ende Juni) hochgerechnet.

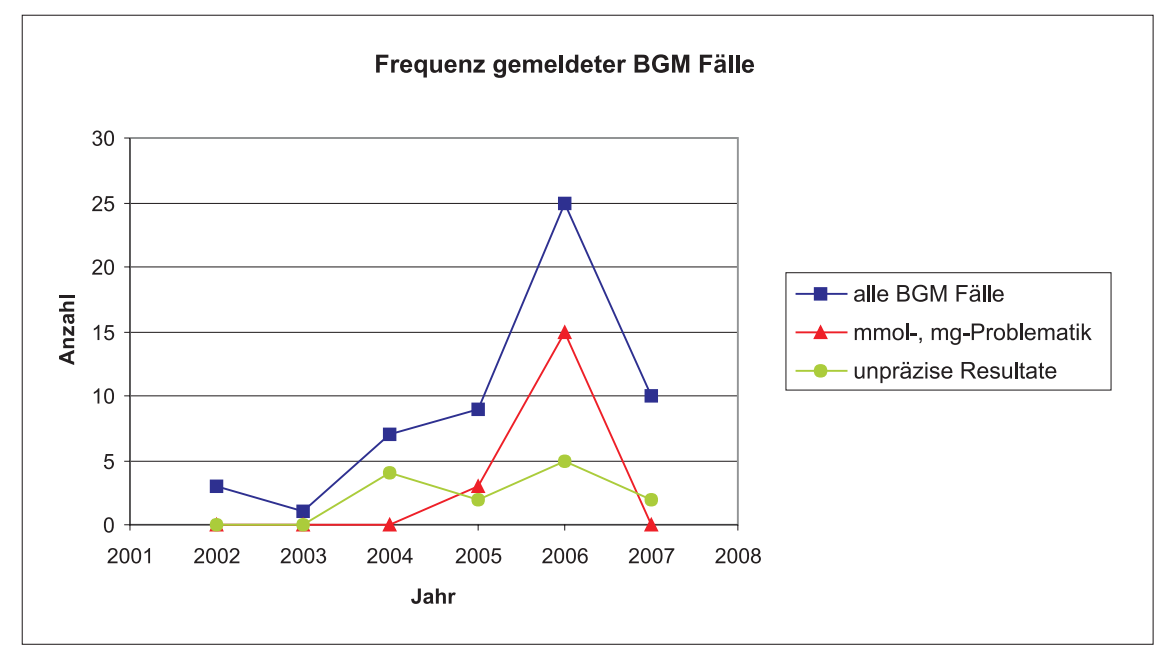

der Materiovigilancemeldungen dazu wurde dieser Umstand als Problem erkannt, und entsprechende Massnahmen konnten eingeleitet werden.

\section{Methode}

Materiovigilancemeldungen zu BGM, die in der Zeitspanne 2002 bis Juni 2007 bei Swissmedic eingegangen sind, wurden retrospektiv analysiert. Die Meldungen wurden aufgrund der erhaltenen Fallbeschreibungen in Problemkreise mit phänomenologisch verwandter Problematik gruppiert. Anhand des zeitlichen Auftretens der Problemkreise wurde auf Häufungen geschlossen.

Die Hersteller, die BGM für Diabetiker in der Schweiz in Verkehr bringen, wurden von Swissmedic kontaktiert und um Stellungnahme zur Problematik der Messeinheiten gebeten. Gleichzeitig wurde nach den in der Schweiz erhältlichen BGM und den Verkaufszahlen gefragt.

\section{Resultate}

\section{Gemeldete Probleme}

In der Zeitspanne vom 1. Januar 2002 bis zum 30. Juni 2007 sind 50 Materiovigilancemeldungen zu BGM bei Swissmedic eingegangen. Anhand der Beschreibungen wurden die Meldungen in 8 Problemkreise gruppiert (Abb. 1). Auffallend war die hohe Anzahl $(\mathrm{n}=18)$ von Meldungen zur Problematik der Messeinheiten (mmol/L und $\mathrm{mg} / \mathrm{dL}$ ). Darunter waren vier Vorkommnisse in der Schweiz, bei denen Patienten nach einer unbeabsichtigten Umstellung der Messeinheit von mmol/L auf mg/dL ihren Blutzuckerwert als zu hoch eingestuft hatten. Diese Fehlinterpretation hatte zur Verabreichung einer Überdosis Insulin geführt, z.T. mit nachfolgender Hypoglykämie. Angaben in $\mathrm{mg} / \mathrm{dL}$ sind $18 \mathrm{mal}$ höher als jene in mmol/L. Wenn ein Wert von $108 \mathrm{mg} / \mathrm{dL}$ Blutglukose (ein normaler Wert) fälschlicherweise für $108 \mathrm{mmol} / \mathrm{L}$ oder $10,8 \mathrm{mmol} / \mathrm{L}$ (ein hoher Wert) gehalten wird, kann dies zur ungewollten Verabreichung von Insulin führen. Der zweitgrösste Problemkreis in der analysierten Zeitspanne umfasst Meldungen zu unpräzisen Messresultaten $(\mathrm{n}=12)$.

\section{Häufung der Problematik mit verschiedenen Messeinheiten}

Die Frequenzen eingegangener Meldungen zu BGM wurden untersucht (Abb. 2). Im Jahr 2006 zeigte sich gegenüber dem Vorjahr ein Anstieg der Gesamtanzahl der Meldungen zu BGM. Deckungsgleich war ein Frequenzanstieg für die Problematik der Messeinheiten zu beobachten. 
Der Anstieg der Gesamtmeldungen zu BGM im Jahr 2006 ergibt sich hauptsächlich aus den Meldungen zur Problematik der Messeinheiten. Im Gegensatz zum Anstieg der Probleme mit Messeinheiten im Jahr 2006 ist für Meldungen zu unpräzisen Resultaten keine Häufung festzustellen.

\section{Marktsituation in der Schweiz}

Es gibt BGM, deren Messeinheit vom Diabetiker selbst umgestellt werden kann, und vermehrt Messgeräte, die auf eine Messeinheit fixiert sind.

Abbildung 3

Materiovigilance-Meldeformular.

\begin{tabular}{|c|c|}
\hline swíssmedic & $\begin{array}{r}\text { Vigilance Medizinprodukte } \\
\text { Melden von Vorkommnissen durch Anwender }\end{array}$ \\
\hline $\begin{array}{l}\text { Herkunft der Meldung } \\
\text { Gesundheitseinrichtung: } \\
\text { Strasse: } \\
\text { PLZ, Ort: } \\
\text { Kontaktperson: } \\
\text { Telefon und Faxnummer: } \\
\text { E-Mail: } \\
\text { Datum der Meldung: }\end{array}$ & $\begin{array}{l}\text { Swissmedic } \\
\text { Abteilung Medizinprodukte } \\
\text { Erlachstrasse } 8 \\
\text { CH-3000 Bern } 9 \\
\text { Fax: } 0313227646 \\
\text { E-Mail: } \\
\text { medical.devices@swissmedic.ch }\end{array}$ \\
\hline \multicolumn{2}{|c|}{ Beschreibung des betroffenen Medizinprodukts } \\
\hline $\begin{array}{l}\text { Lieferant (Name, Ortschaft): } \\
\text { Kontaktperson (Lieferant/Herst }\end{array}$ & Hersteller (Name): \\
\hline Handelsname des Produkts: & Art des Produkts (z.B. Herzschrittmacher, Katheter): \\
\hline Modell- oder Katalognummer: & Serien- oder Losnummer: \\
\hline
\end{tabular}

Vigilance Medizinprodukte

Beschreibung des Zwischenfalls
Datum des Zwischenfalls:
Falls relevant: Geburtsjahr des Patienten: $\quad$ Geschlecht: $\square \mathrm{m} \quad \square \mathrm{f}$
Beschreibung des Zwischenfalls und der Folgen für den Patienten:

Beschreibung des Zwischenfalls und der Folgen für den Patienten:

Ich habe den Zwischenfall bereits an die Kontaktperson (Hersteller/Lieferant) gemeldet: Swissmedic darf diese Angaben für eine Untersuchung an den Hersteller weiterleiten:

Swissmedic - Erlachstrasse 8 - CH-3000 Bern 9 - www.swissmedic.ch - Tel, +413132322 51 - Fax +4131322 7646
Swissmedic hat die Marktsituation für BGM in der Schweiz in Zusammenarbeit mit den Herstellern abgeklärt. Es wurden sieben Hersteller kontaktiert, die in der Schweiz BGM anbieten. Im Frühling 2006 waren in der Schweiz gesamthaft 160965 BGM in Verkehr. Davon wurden 99,6\% von vier Firmen vertrieben. Der Anteil der umstellbaren Geräte betrug zu diesem Zeitpunkt noch $25 \%$.

\section{Diskussion}

Sofern die relevanten Zwischenfälle gemeldet werden, kann das Materiovigilancesystem Gefährdungen erkennen und darauf reagieren. Voraussetzung dafür ist, dass die am System beteiligten Parteien ihre Meldepflicht wahrnehmen. Gegenüber der Überwachungsbehörde meldepflichtig sind die Hersteller bzw. Händler sowie Fachpersonen. Dazu gehören insbesondere Spitalpersonal, praktizierende Ärzte, aber auch Fachpersonen in Apotheken und Drogerien.

Wenn Fachpersonen in der Schweiz bei der Anwendung von Medizinprodukten ein schwerwiegendes Vorkommnis feststellen, müssen sie Swissmedic eine entsprechende Meldung zustellen. Für die Meldung steht ein einfaches Formular (Abb. 3) zur Verfügung [3]. Meldepflichtig sind schwerwiegende Vorkommnisse mit Medizinprodukten, d.h., wenn wegen eines Medizinproduktes der Tod oder eine schwerwiegende Beeinträchtigung des Gesundheitszustandes von Patienten, Anwendern oder von Dritten eingetreten ist oder hätte eintreten können. Swissmedic leitet die Meldung an den Hersteller weiter und überwacht dessen Untersuchung. Die Fachperson, die die Meldung an Swissmedic erstellt hat, wird über den Ausgang der Untersuchung informiert. Sofern der Hersteller Korrekturen am Medizinprodukt einleitet, überwacht Swissmedic deren Durchführung. Nicht jede Meldung von Fachpersonen führt zu Korrekturmassnahmen, da nicht jedes eintretende Vorkommnis auf ein fehlerhaftes Medizinprodukt zurückzuführen ist. Die Meldepflicht für Fachpersonen besteht seit 2002. Die bisherigen Erfahrungen für alle Medizinprodukte haben gezeigt, dass 20-25\% der von Fachpersonen gemeldeten Zwischenfälle zu einer Korrektur an Medizinprodukten geführt haben. Isolierte Einzelfälle müssen nicht auf ein generelles Problem hindeuten. Wenn sich aber die Meldungen häufen, werden Massnahmen erwogen.

Die Meldungen zur Problematik mit den BGM-Messeinheiten haben zu Korrekturmassnahmen geführt. Einige Gerätehersteller haben Diabetiker und Diabetesfachleute schriftlich 
über das Risiko informiert. Weiter bieten die Hersteller vermehrt fixierte Geräte an, die nicht mehr unbeabsichtigt umgestellt werden können. Damit wurde das Risiko einer unbeabsichtigten Umstellung der Messeinheit gesenkt.

Swissmedic veröffentlicht im Internet die Korrekturmassnahmen der Hersteller, die für Anwender in der Schweiz relevant sind [4]. In dieser Veröffentlichung sind verschiedene Warnschreiben zu BGM zu finden, u.a. zur Problematik der Messeinheiten. Sofern notwendig, kann Swissmedic auch weiter gehende Empfehlungen ausarbeiten und veröffentlichen. Dies ist für die Problematik der Messeinheiten von BGM erfolgt [5]. Sofern angezeigt, kann Swissmedic auch eine spezifische Information in entsprechenden Zeitschriften anstreben, was im Fall der BGM mit einer Veröffentlichung im D-Journal, einer Zeitschrift für Diabetiker, verwirklicht wurde [6].

Das Beispiel der BGM und der Problematik mit den Messeinheiten zeigt, dass sich mit dem Materiovigilancesystem Produktrisiken erkennen lassen. Durch eine gezielte Reduktion dieser Risiken lässt sich die Patientensicherheit erhöhen. Eine wichtige Voraussetzung für das Funk- tionieren des Materiovigilancesystems ist, dass die professionellen Anwender schwerwiegende Vorkommnisse mit Medizinprodukten konsequent melden, sowohl beim Hersteller als auch bei Swissmedic. Swissmedic ruft daher insbesondere Ärzte auf, ihre Beobachtungen über schwerwiegende Vorfälle mit Geräten, Instrumenten und anderen medizinischen Hilfsmitteln an Swissmedic zu melden.

\section{Literatur}

1 Medizinprodukteverordnung MepV, SR 812.213.

2 Schlegel A. Materio-Vigilance Meldungen durch Labors. Pipette - Swiss Laboratory Medicine. 2005; 1:20-2.

3 Abrufbar unter: www.swissmedic.ch/md. asp\#vigilance.

4 Veröffentlichung unter: www.swissmedic.ch/ md.asp\#recalls.

5 Blutzuckermessgeräte für Diabetiker: Achtung bei der Messeinheit! Swissmedic Journal. 2006; 9:919-21.

6 Scheidegger K. mg/dl oder mmol/l? Passen Sie auf! D-Journal. 2006;182:23. 